\title{
Predictions on the total cross section and real part at LHC and SSC
}

\author{
UA4/2 Collaboration \\ Genova-Palaiseau-Praha-Roma-Valencia
}

C. Augier ${ }^{b}$,J. Bourotte ${ }^{b}$, M. Bozzo ${ }^{a}$, A. Bueno ${ }^{e}$, R. Cases ${ }^{e}$,

F. Djama ${ }^{e}$, M. Haguenauer ${ }^{b}$, V. Kundrát ${ }^{c}$, M. Lokajíček ${ }^{c}$,

G. Matthiae ${ }^{d}$, A. Morellia ${ }^{a}$ F. Natalid ${ }^{d}$, S. Němeček ${ }^{c}$, M. Novák ${ }^{c}$,

E. Sanchis ${ }^{e}$, G. Sette ${ }^{a}$, M. Smižanskác ${ }^{c}$ J. Velasco ${ }^{e}$

${ }^{a}$ Università di Genova and Sezione INFN, Genova, Italy

${ }^{b}$ Ecole Polytechnique/IN2P3-CNRS, Palaiseau, France

${ }^{c} A V \breve{C} R$, Institute of Physics, Praha, Czech Republic

${ }^{d}$ Università di Roma II and Sezione INFN, Roma, Italy

${ }^{e}$ IFIC-Centro Mixto Universidad de Valencia-CSIC, Valencia, Spain

\begin{abstract}
An overall fit to the data on the total cross section and on the real part of the forward amplitude for $\bar{p} p$ and $p p$ was performed using dispersion relations. The energy dependence of the total cross section was studied up to $\sqrt{s}=100$ TeV. Numerical predictions are presented for LHC and SSC energies.
\end{abstract}

\section{GENEVA}

1993

Submitted to Phys. Lett. B 

The discovery of the rising total cross sections at the CERN ISR was followed, after more than a decade, by new measurements at the S̄ppS and Tevatron colliders which definitely proved that cross sections are still rising in the TeV energy region. However, what actually is the energy dependence of the cross section in this high-energy domain is a much discussed question which still remains without a definite answer.

In fact existing models make different predictions. The impact picture model [1] predicts a $(\ln s)^{2}$ dependence which asymptotically will saturate the Froissart-Martin bound [2], while Regge pole models [3] exhibit a power law, $s^{\alpha(0)-1}$, with an intercept $\alpha(0)$ of the Pomeron trajectory which is slightly above unit. Both models reproduce present data in a satisfactory way.

On the other hand, it is well known that the energy dependence of the scattering amplitude in the forward direction can be investigated independently of theoretical models using the dispersion relations which are based only on fundamental principles. It is customary to describe the forward amplitude in terms of the total cross section $\sigma_{\text {tot }}$ which, by virtue of the optical theorem, is proportional to the imaginary part and of the parameter $\rho$ which is defined as the ratio of the real to the imaginary part.

This approach was pioneered by Amaldi et al. [4] who fitted data on $\sigma_{\text {tot }}$ and $\rho$ in the energy interval $5<\sqrt{s}<62 \mathrm{GeV}$, with dispersion relations. They predicted that the total cross section would be in the range from 55 to $70 \mathrm{mb}$ at the energy of the $\mathrm{S} \overline{\mathrm{p} p S}$ Collider. The measurement [5] gave the result $\sigma_{\text {tot }}=62.2 \pm 1.5 \mathrm{mb}$, which is well within these limits, thus confirming the validity of the method.

In this paper we present a similar analysis. We performed a simultaneous fit on $\sigma_{\text {tot }}$ and $\rho$ for $\mathrm{pp}$ and $\overline{\mathrm{p}} \mathrm{p}$ scattering which provides predictions on the behaviour of the total cross section up to $\sqrt{s} \simeq 100 \mathrm{TeV}$. We remark that the dispersion relations provide a general framework which may also be used to check the consistency among different sets of data.

Assuming that the odd under crossing forward amplitude becomes negligible at very high energy, we can use the standard (once subtracted) 
dispersion relations [6],

$$
\rho_{ \pm}(E) \sigma_{ \pm}(E)=\frac{B}{p}+\frac{E}{\pi p} \int_{m}^{\infty} d E^{\prime} p^{\prime}\left[\frac{\sigma_{ \pm}\left(E^{\prime}\right)}{E^{\prime}\left(E^{\prime}-E\right)}-\frac{\sigma_{\mp}\left(E^{\prime}\right)}{E^{\prime}\left(E^{\prime}+E\right)}\right]
$$

Where $E$ is the energy of the incoming particle in the lab sytem and $p$ its momentum, $m$ is the proton mass and $B$ is the substraction constant. The index + or - refers to $\mathrm{pp}$ and $\overline{\mathrm{p}} \mathrm{p}$ respectively. To evaluate the integral in eq. 1 an analytic expression as a function of the laboratory energy is needed for the total cross section. Here, we have adopted the same parametrization as in ref.(4)

$$
\sigma_{ \pm}(E)=A_{1} E^{-N_{1}} \mp A_{2} E^{-N_{2}}+\sigma_{\infty}
$$

Where $A_{1} E^{-N_{1}}$ and $A_{2} E^{-N_{2}}$ are Regge-type terms which describe the behaviour at low energy and the difference between $\sigma_{\bar{p} p}$ and $\sigma_{p p}$. We assume that at high-energy the cross section varies as a power of $\ln s$,

$$
\sigma_{\infty}=C_{0}+C_{2}\left(\ln s / s_{0}\right)^{\gamma}
$$

where the scale factor $s_{0}$ is taken equal to $1 \mathrm{GeV}^{2}$.

In view of the reported discrepancy between the measurements of the total cross section at the Tevatron Collider [7], [8], we have restricted the data sample on which our dispersion relation fit is performed, to the energy interval $5<\sqrt{s}<546 \mathrm{GeV}$. In the present analysis, therefore, the data on total cross section at $\sqrt{s}=546 \mathrm{GeV}[5],[8]$ and the recent measurement of $\rho$ at the same energy [9] will play an important role in the determination of the extrapolation to higher energies.

The eight parameters $A_{1}, N_{1}, A_{2}, N_{2}, C_{0}, C_{2}, \gamma$ and $B$ are determined by a $\chi^{2}$ minimization procedure which is performed on a set of 103 data points. In eqs. 1 to 3 , the cross section is measured in $\mathrm{mb}$ and energy in $\mathrm{GeV}$. The values of the parameters as given by the best fit $\left(\chi^{2} /\right.$ d.o.f. $\left.=78.5 / 95\right)$ are shown in Table I where the quoted errors correspond to an increase of the $\chi^{2}$ by one unit. 


\begin{tabular}{|c|c|}
\hline$A_{1}=42.5_{-1.6}^{+2.0}$ & $N_{1}=0.45_{-0.06}^{+0.08}$ \\
\hline$A_{2}=25.5_{-0.4}^{+0.5}$ & $N_{2}=0.565_{-0.004}^{+0.005}$ \\
\hline$C_{0}=30 ._{-4 .}^{+3 .}$ & $C_{2}=0.10_{-0.06}^{+0.15}$ \\
\hline$\gamma=2.25_{-0.31}^{+0.35}$ & $B=-57 . \pm 4$. \\
\hline
\end{tabular}

Table I. Values of the parameters of our best fit

The large errors on $C_{2}$ and $\gamma$ are induced by the strong correlation existing between these two parameters. The result of our best fit and the interval of uncertainty corresponding to one standard deviation are shown in Fig.1 and 2 together with the experimental data. In Table $I I$ we have collected numerical results given by the best fit at the energy of the existing colliders and of the future machines, LHC $(\sqrt{s}=16 \mathrm{TeV})$, and SSC $(\sqrt{s}=40 \mathrm{TeV})$. Available experimental data [5],[7],[8],[9],[10], are also listed in Table II for comparison.

\begin{tabular}{|c|c|c|c|}
\hline$\sqrt{s}(\mathrm{TeV})$ & & $\sigma_{\text {tot }}(\mathrm{mb})$ & $\rho$ \\
\hline 0.55 & Fit & $61.8 \pm 0.7$ & $0.138 \pm 0.010$ \\
& UA4 & $62.2 \pm 1.5$ & $0.135 \pm 0.015$ \\
& CDF & $61.5 \pm 1.0$ & \\
\hline 0.90 & Fit & $67.5 \pm 1.3$ & $0.141 \pm 0.012$ \\
& UA5 & $65.3 \pm 1.7$ & \\
\hline 1.8 & Fit & $76.5 \pm 2.3$ & $0.142 \pm 0.015$ \\
& E710 & $72.8 \pm 3.1$ & $0.140 \pm 0.069$ \\
& CDF & $80.6 \pm 2.3$ & \\
\hline 16. & Fit & $111 . \pm 8$. & $0.133 \pm 0.018$ \\
\hline 40. & Fit & $130 . \pm 13$. & $0.127 \pm 0.019$ \\
\hline
\end{tabular}

Table II. Results of our best fit and experimental data

The experimental results at $\sqrt{s}=0.55 \mathrm{TeV}$ are well reproduced by the best fit. At $\sqrt{s}=1.8 \mathrm{TeV}$, our fit predicts a value of the total cross section which lies in between the two measurements which have been reported [7], [8]. 
In spite of the large error on the parameter $\gamma$ as derived from the best fit, our analysis favours a $(\ln s)^{2}$ dependence of the total cross section in the multi TeV region with respect to a $\ln s$ one. This behaviour has been often referred to as a "qualitative" saturation of the Froissart-Martin bound in the sense that it corresponds to the maximum rate of rise with energy which is allowed by analyticity and unitarity, while numerically actual data lie much below the bound itself.

In order to see more clearly how much the data are sensitive to the power of the ln $s$ term, we have performed a fit by fixing the parameter $\gamma$ to the value $\gamma=1$. The result of this fit which has $\chi^{2} /$ d.o.f. $=82.0 / 96$ is shown in Fig. 3 together with the best fit and the experimental data on $\sigma_{\text {tot }}$. Recent cosmic rays results [11] from the Akeno Observatory obtained from the analysis of proton-air interactions at ultrahigh energies are also plotted in Fig.3. They are well reproduced by our best fit. 


\section{REFERENCES}

[1] H.Cheng and T.T.Wu, Phys. Rev. Lett. 24 (1970) 1456

C.Bourrely et al., Z. Phys. C37 (1988) 369.

[2] M.Froissart, Phys. Rev. 123 (1961) 1053

A.Martin, Nuovo Cimento 42 (1966) 930; ibid. 44 (1966) 1219

[3] A.Donnachie and P.Landshoff, Nucl. Phys. B267 (1986) 690 and Phys. Lett. B296 (1992) 227

R.M.J.Covolan et al., Nucl. Phys.B (Proc. Suppl.) 25B (1992) 86.

[4] U.Amaldi et al., Phys. Lett. B66 (1977) 390

[5] UA4 Collab., M.Bozzo et al., Phys. Lett. B147 (1984) 392

[6] P.Söding, Phys. Lett. 8 (1964) 285.

[7] E710 Collab., N.A.Amos et al., Phys. Rev. Lett. 68 (1992) 2433

[8] CDF Collab. Paper presented by P.Giromini at the 5th Blois Workshop on Elastic and Diffractive Scattering, Brown University, Providence, RI, USA, (June 1993).

[9] UA4/2 Collab., C.Augier et al., CERN-PPE / 93-115 preprint, submitted to Phys. Lett. B

[10] UA5 Collab., G.J.Alner et al., Z. Phys. C32 (1986) 153.

[11] M.Honda et al., Phys. Rev. Lett. 70 (1993) 525 


\section{FIGURE CAPTIONS}

Fig. 1 The result of our fit is shown together with the experimental data on $\sigma_{p p}$ and $\sigma_{\bar{p} p}$ from accelerators. The best fit is represented by the solid line while the region of uncertainty corresponding to one standard deviation is delimited by the two dashed lines.

Fig. 2 The result of the fit is shown together with the experimental data on the parameter $\rho$. Solid and dashed lines have the same meaning as in Fig.1.

Fig. 3 Total cross section data from accelerators and from cosmic rays are shown together with our best fit (solid line), the region of uncertainty (delimited by the two dashed lines) and the fit obtained with $\gamma=1$ (dotted line). 
Figure 1

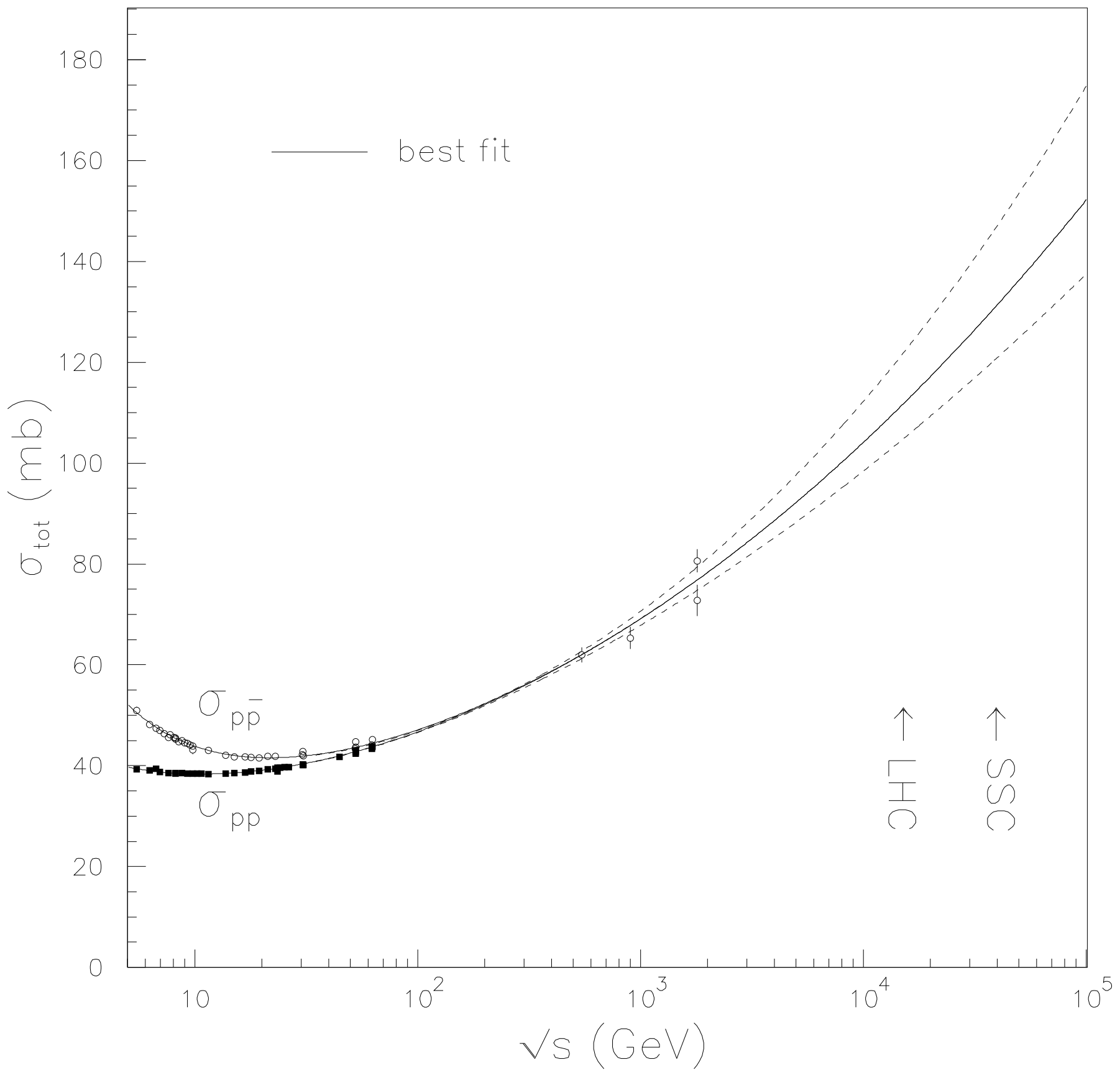


Figure 2

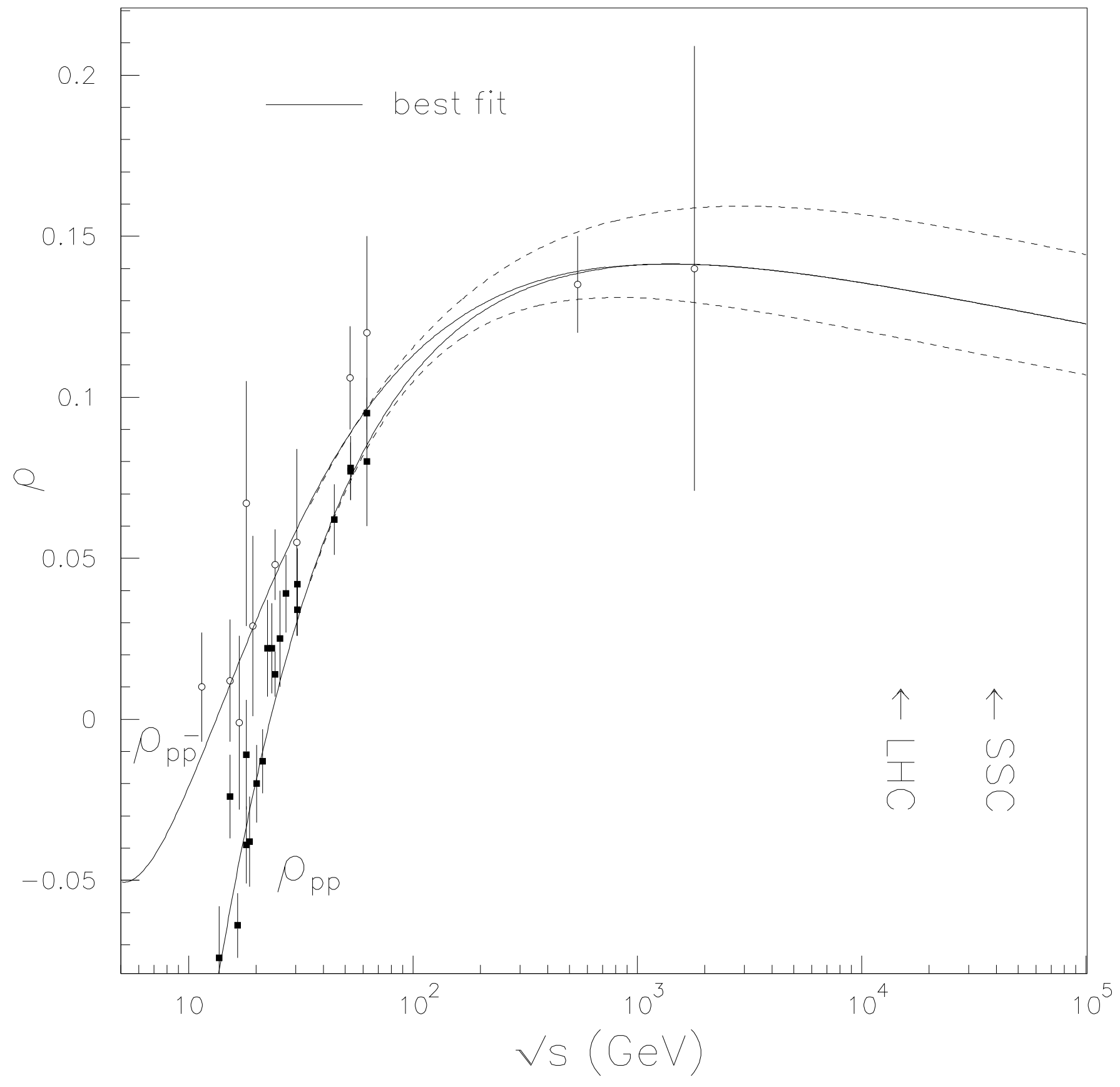


Figure 3

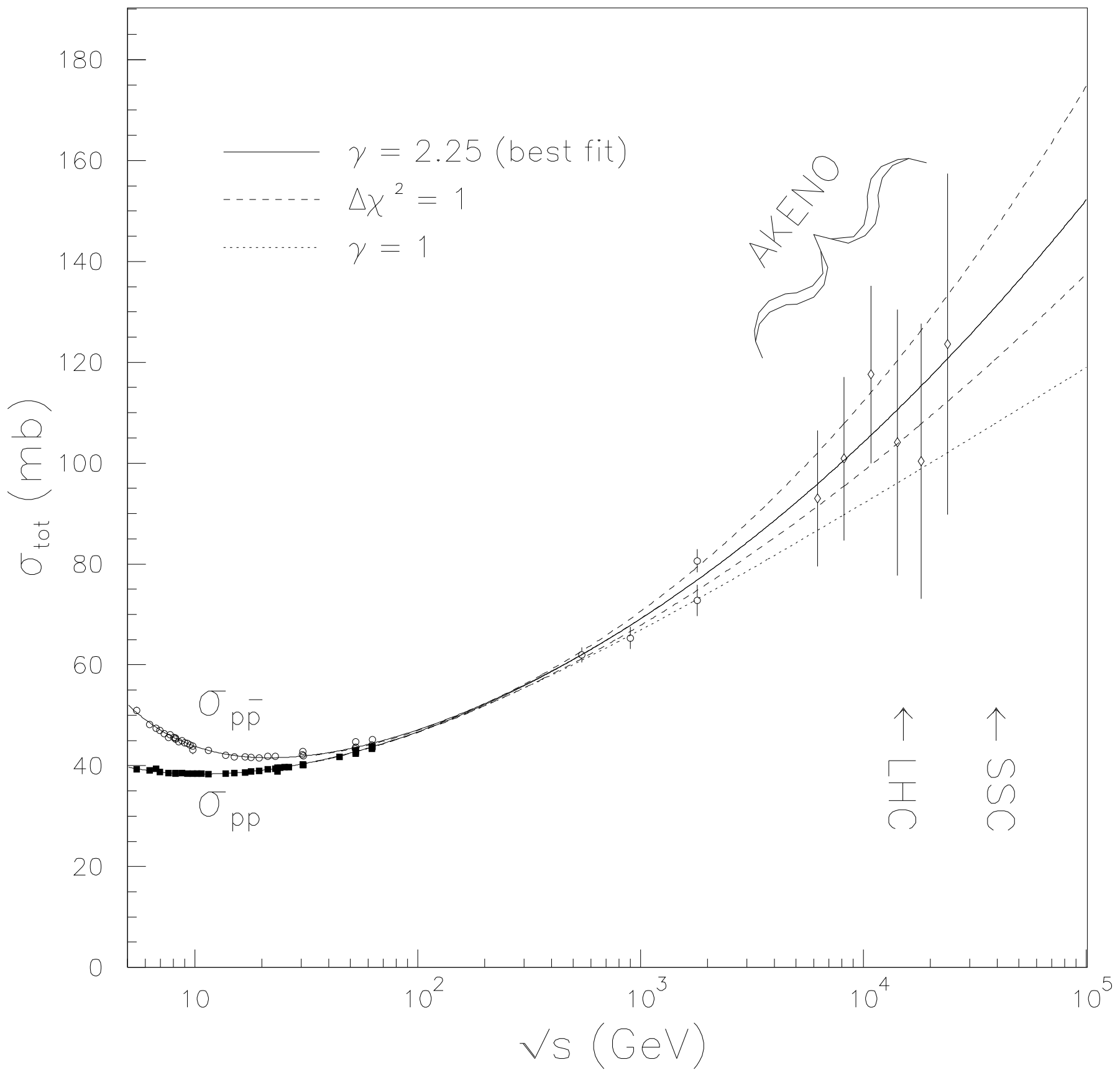

